\title{
Manifestação cardiovascular na infecção por COVID-19 em paciente com artrite reumatóide: relato de caso
}

\author{
Cardiovascular manifestation in COVID-19 infection in a patient with rheumatoid arthritis: case \\ report
}

Manifestación cardiovascular en infección por COVID-19 en paciente con artritis reumatoide: reporte de caso

\section{Resumo}

Este estudo teve como objetivo relatar o caso de uma paciente do sexo feminino, 65 anos, com diagnóstico prévio de artrite reumatoide, dentre outras comorbidades que corroboram para o aumento do risco cardiovascular, atendida no ano de 2020 em hospital particular de Belém, devido ao quadro de síndrome coronariana aguda associada à infecção pelo vírus SARS-Cov-2. Devido ao perfil clínico, a paciente foi submetida a eletrocardiograma que evidenciou infarto do miocárdio com alteração de supra do segmento ST, indicando a necessidade de terapia de reperfusão, oxigenioterapia e monitorização de sinais vitais. Apresentou tomografia de tórax evidenciando padrão de "vidrofosco" e comprometimento de mais de $25 \%$ do parênquima pulmonar e evoluiu com trombose de artéria femoral. Após tratamento em unidade de terapia intensiva, recebeu alta do serviço de saúde, mantendo-se estável no ponto de vista reumatológico, cardiovascular e pulmonar.

Palavras-chave: Idosos; Doenças cardiovasculares; COVID-19.

\begin{abstract}
This study aimed to report the case of a 65 -year-old female patient with a previous diagnosis of rheumatoid arthritis, among other comorbidities that corroborate the increase in cardiovascular risk, seen in 2020 in a private hospital in Belém, due to picture of acute coronary syndrome associated with SARS-Cov-2 virus infection. Due to the clinical profile, the patient underwent an electrocardiogram that showed myocardial infarction with ST-segment elevation alteration, indicating the need for reperfusion therapy, oxygen therapy and monitoring of vital signs. A chest CT scan showed a "ground glass" pattern and involvement of more than $25 \%$ of the lung parenchyma and evolved with thrombosis of the femoral artery. After treatment in the intensive care unit, he was discharged from the health service, remaining stable from a rheumatological, cardiovascular and pulmonary point of view.
\end{abstract}

Keywords: Seniors; Cardiovascular diseases; COVID-19. 


\begin{abstract}
Resumen
Este estudio tuvo como objetivo reportar el caso de una paciente de 65 años con diagnóstico previo de artritis reumatoide, entre otras comorbilidades que corroboran el aumento del riesgo cardiovascular, atendida en 2020 en un hospital privado de Belém, por cuadro de cuadro agudo. Síndrome coronario asociado con la infección por el virus del SARS-Cov-2. Debido al perfil clínico, el paciente fue sometido a un electrocardiograma que mostró un infarto de miocardio con alteración de la elevación del segmento ST, lo que indica la necesidad de terapia de reperfusión, oxigenoterapia y monitorización de constantes vitales. Una tomografía computarizada de tórax mostró un patrón en "vidrio esmerilado" y afectación de más del 25\% del parénquima pulmonar y evolucionó con trombosis de la arteria femoral. Tras el tratamiento en la unidad de cuidados intensivos, fue dado de alta del servicio de salud, manteniéndose estable desde el punto de vista reumatológico, cardiovascular y pulmonar.
\end{abstract}

Palabras clave: Mayores; Enfermedades cardiovasculares; COVID-19.

\title{
1. Introdução
}

A doença de coronavírus 2019 (COVID-19) evoluiu rapidamente para uma pandemia mundial. Causada por síndrome respiratória aguda grave de coronavírus (SARS-CoV-2) (Aviña-Zubieta et al., 2013). Com base na filogenia e taxonomia, o SARS-CoV-2 foi classificado pelo Comitê Internacional de Taxonomia de Vírus (ICTV) como um vírus pertencente ao gênero Betacoronavirus, subfamília Coronavirinae, família Coronaviridae, ordem Nidovirales, reino Riboviria (Zhu et al., 2020). O agravo impactou milhões de vidas e contribuiu para um número crescente de mortes em todo o mundo (Mikuls et al., 2020).

Foram confirmados no mundo 96.267.473 casos de COVID-19 (624.353 novos em relação ao dia anterior) e 2.082.745 mortes (16.079 novas em relação ao dia anterior) até 22 de janeiro de 2021 (Who, 2021). No mesmo período, na Região das Américas, 25.738.091 pessoas que foram infectadas pelo novo coronavírus se recuperaram, porém 983.878 pessoas foram a óbito.

Enquanto que no Brasil, segundo a Organização Mundial da Saúde, foram confirmados 8.753.920 casos (56.552 novos em relação ao dia anterior) e 215.243 mortes (1096 novas em relação ao dia anterior) até 24 de janeiro de 2021 (Opas, 2021).

Em sua última atualização, o Centers for Disease Control and Prevention (CDC) (Who, 2019) definiu que o SARSCoV-2 pode se manifestar em quatro categorias de gravidade, sendo a leve observada naqueles pacientes que apresentam qualquer um dos sinais e sintomas (febre, tosse, mal estar e dor muscular), não estando associados à falta de ar, dispneia ou imagens torácicas anormais, até a categoria de doença crítica, na qual o paciente pode vir a apresentar insuficiência respiratória, choque séptico e/ou disfunção de múltiplos órgãos.

Os estudos foram incessantes no que se relaciona ao acometimento cardiovascular frente à infecção pelo SARS-CoV2. Uma análise dos estudos de Wuhan evidenciou letalidade geral de 2,3\%, embora em indivíduos com doença cardiovascular essa taxa possa chegar a 10,5\% (CDC, 2020). A injúria miocárdica ocorre em $7 \%$ a $20 \%$ dos pacientes com COVID-19, podendo ser atribuída à gravidade da doença, miocardite ou infarto agudo do miocárdio (IAM) (Filippini et al., 2020). Desse modo, o observado até então é a estreita relação da COVID-19 com a lesão cardíaca em pacientes hospitalizados (Fernández et al., 2020). A hipoxemia se relaciona de forma indireta com a lesão dos cardiomiócitos, bem como as citocinas inflamatórias em alta geram uma maior tendência de episódios trombóticos em pacientes com predisposição. Dessa maneira, destaca-se a importância de estratificar o risco e antecipar a necessidade de terapias avançadas nos pacientes acometidos por essa doença.

No que diz respeito à associação de doenças reumáticas e a COVID-19, as características clínicas entre pacientes com doenças reumáticas sistêmicas são variáveis. Há dados insuficientes para determinar se o tipo de doença reumática ou o uso de agentes imunossupressores utilizados influenciam na apresentação clínica da COVID-19 nessa população. No entanto, é possível que algumas características da doença reumática possam ser difíceis de distinguir da COVID-19 (Mikuls et al., 2020). 
A artrite reumatoide é uma doença sistêmica autoimune de causa desconhecida caracterizada principalmente pela presença de inflamação sinovial, lesão cartilaginosa e deformidade articular, além de estar associada a inúmeras comorbidades. A doença cardiovascular (DCV) é a principal causa de mortalidade nos pacientes com artrite reumatoide (AR) (McInnes \& Scheet, 2011) e o IAM é o evento mais comum (Bergström \& Turesson, 2009; Solomon et al., 2010).

Atualmente não há evidências que correlacionem fatores de risco para resultados ruins em pacientes com a COVID-19 e doença reumática (Wu, 2020; Zhou et al., 2020). Com base em estudos de coorte retrospectivos (Yang et al., 2020; Richardson et al., 2020) os fatores de risco para complicações na infecção por COVID-19 incluem idade mais avançada (> 65 anos) e comorbidades selecionadas, como doença pulmonar crônica, hipertensão arterial sistêmica, doença arterial coronariana, doença renal crônica, obesidade e diabetes mellitus, condições frequentemente presentes em pacientes com doença reumática (Nurmohamed et al., 2015; Bichile \& Petri, 2014).

Diante disso, o objetivo do presente relato de caso é descrever o caso clínico de uma paciente idosa com diagnóstico prévio de artrite reumatoide que apresentou comprometimento cardiovascular após infecção por COVID-19.

\section{Metodologia}

Trata-se de uma pesquisa exploratória, descritiva, de abordagem qualitativa, baseada nos preceitos de (Gomes, 2008). A coleta de dados foi realizada diretamente via prontuário semiestruturado da paciente associado a consultas ambulatoriais de um hospital de referência na cidade de Belém, capital do estado do Pará.

Este estudo obedeceu a todas as normas e preceitos éticos, atendendo a resolução 466/12/CONEP, conforme aprovação pelo Comitê de Ética e Pesquisa em Seres Humanos do Instituto de Ciências da Saúde da Universidade Federal do Pará sob (CAAE): 31800720.1.1001.0018.

\section{Relato de Caso}

Paciente do sexo feminino, 65 anos, com diagnósticos prévios de artrite reumatoide há mais de 20 anos, hipertensão arterial sistêmica, hipotireoidismo, dislipidemia e obesidade grau III, usuária crônica de corticoide oral e intramuscular, em uso de prednisona $10 \mathrm{mg} / \mathrm{dia}$, metotrexato $20 \mathrm{mg} / \mathrm{semana}$, levotiroxina $50 \mathrm{mcg} / \mathrm{dia}$, sinvastatina $40 \mathrm{mg} / \mathrm{dia}$, losartana $100 \mathrm{mg} / \mathrm{dia}$ e anlodipino $5 \mathrm{mg} / \mathrm{dia}$, deu entrada, em julho/2020, no pronto atendimento hospitalar, às 5:30h, com quadro de sudorese noturna há uma semana e dor em região precordial tipo aperto com irradiação para dorso, de caráter súbito, associado a sudorese profusa, diarreia, náuseas e vômitos iniciados as 4:00h. Fez uso de escopolamina 10mg, carvedilol 25mg e ácido acetil salicílico $100 \mathrm{mg}$ por conta própria.

Durante admissão, paciente consciente, orientada, bradicárdica, oximetria e pressão arterial sem alterações, sendo submetida a eletrocardiograma que evidenciou infarto agudo do miocárdio com supradesnivelamento do segmento ST em parede inferior. Realizou coleta de troponina, CPK e CKMB, de forma seriada, com alteração ascendente dos três marcadores de necrose miocárdica. Administrado AAS, clopidogrel, enoxaparina e oxigênio por cateter nasal devido síndrome coronariana aguda.

Após 7 horas do quadro inicial, foi encaminhada para realização de cateterismo por diagnóstico de infarto agudo do miocárdio (IAM) com supradesnivelamento de ST em parede inferior, com identificação de obstrução de artéria coronária direita por trombo com migração para a região distal do vaso e não passível de angioplastia. Imediatamente após fim do procedimento, paciente evoluiu com queixa de dor intensa em região de membro inferior esquerdo. Devido quadro, foi encaminhada para realização de tomografia computadorizada (TC) de tórax por suspeita clínica de COVID-19 e angiotomografia de abdome e pelve, para investigação de possível trombose arterial de membro inferior esquerdo devido dor 
súbita local.

Indicado tratamento antitrombótico com cloridrato de tirofibana e anticoagulação plena com enoxaparina. TC de tórax apresentou vidro fosco em bases, com comprometimento de $25 \%$ do parênquima pulmonar, compatível com diagnóstico de COVID-19. Realizada dosagem de D-dímero, cujo valor foi 4 vezes superior ao limite de referência. Ademais, foi identificado trombose da artéria ilíaca esquerda por meio de angiotomografia, sendo a paciente submetida à embolectomia em caráter de urgência. Posteriormente, foi encaminhada ao Centro de Terapia Intensiva (CTI) e iniciado tratamento com metilprednisolona endovenosa, ceftriaxona, azitromicina, ácido acetil salicílico, ticagrelor e enoxaparina 60mg/dia. Evoluiu satisfatoriamente, realizando extubação no dia posterior ao procedimento cirúrgico, sem intercorrências.

Realizado RT-PCR- molecular para COVID-19 no terceiro dia de internação hospitalar (DIH) com resultado negativo. Evoluindo com melhora progressiva até desmame completo da oxigenioterapia no $4^{\circ}$ pós-operatório cirúrgico. Frente a isso, apresentou diversos hematomas pelo corpo, edema importante em membro inferior esquerdo e hematúria pela sonda vesical de demora.

Realizou metilprednisolona IV por 5 dias, sendo posteriormente prescrito prednisona $60 \mathrm{mg} / \mathrm{dia}$. Foi encaminhada à enfermaria no $6^{\circ} \mathrm{DIH}$, com diurese espontânea e sem hematúria, respiração em ar ambiente, estável hemodinamicamente.

Recebeu alta hospitalar no $9^{\circ}$ dia de internação hospitalar, após término de antibioticoterapia, sendo feito troca de enoxaparina por rivaroxabana. Após 14 dias do início dos sintomas de síndrome coronariana aguda, paciente realizou teste rápido para COVID-19 oferecido pelo sistema único de saúde de Belém, apresentando amostra reagente para SARS-CoV-2.

Após 1 mês de alta hospitalar, foi realizado ecocardiograma sem alterações significativas e TC de tórax demonstrando áreas de fibrose em base de ambos pulmões.

Atualmente encontra-se estável do ponto de vista cardiovascular, pulmonar e reumatológico, mantendo apenas Ddímero alterado (em 13/12/2020) de $696 \mathrm{ng} / \mathrm{ml}$ (referência inferior a 200ng/ml FEU). Em uso de prednisona 7,5mg/dia, metotrexato $20 \mathrm{mg} / \mathrm{dia}$, leflunomida $20 \mathrm{mg} / \mathrm{dia}$, levotiroxina $50 \mathrm{mcg} / \mathrm{dia}$, rivaroxabana $10 \mathrm{mg} / \mathrm{dia}$, clopidogrel $75 \mathrm{mg} / \mathrm{dia}$, nebivolol $5 \mathrm{mg} /$ dia e corticoide inalatório devido tosse crônica.

\section{Discussão}

A pandemia da COVID-19, causada pela infecção do SARS-CoV-2, impactou milhões vidas e contribuiu para um número crescente de mortes em todo o mundo (Mikuls et al., 2020).

As consequências do vírus SARS-CoV-2 para o miocárdio vem sendo elucidadas, mas ainda há muitas dúvidas (Mikuls, 2003; Burner \& Rosenthal, 2009). Até o momento, compreende-se que a lesão miocárdica se fundamenta na ação da hipoxemia e no estado hiperinflamatório naqueles pacientes que apresentam predisposição a eventos tromboembólicos (Figueiredo et al., 2020).

Situações de grave estresse fisiológico como sepse e insuficiência respiratória presentes em pacientes com COVID-19 estão associadas a elevações de biomarcadores de lesão miocárdica, determinando pior prognóstico. O mecanismo mais provável é um desequilíbrio entre oferta e demanda de oxigênio, podendo haver a ruptura da placa ateromatosa (Sarkisian et al., 2016).

Apesar da paciente não apresentar teste sorológico positivo para COVID-19, foi diagnosticada e tratada de acordo com as recomendações do Ministério da Saúde (Lazaros \& Dimitrios, 2015), que orienta que caso de COVID-19 não necessariamente precisa apresentar teste laboratorial positivo, desde que o paciente apresente clínica suspeita e imagem tomográfica característica de lesão causada pelo novo coronavírus. Neste caso deve-se notificar o caso, assim como iniciar tratamento específico para condição clínica apresentada, em busca de evitar a evolução para síndrome respiratória aguda com ou sem comprometimento cardiovascular. 
Pacientes com artrite reumatoide apresentam risco aumentado de doença cardiovascular, que geralmente ocorre uma década antes, quando em comparação com controles pareados por idade e sexo e possuem duas vezes mais probabilidade de desenvolver infarto do miocárdio independentemente da idade (Lazaros \& Dimitrios, 2015). As razões para o aumento do risco de DCV em pacientes com AR são complexas e postuladas como relacionadas a mecanismos autoimunes e inflamatórios crônicos, disfunção endotelial e gestão inadequada de fatores de risco modificáveis e, potencialmente, a medicamentos, incluindo corticosteroides e antinflamatórios não esteroides (Gabriel, 2012; Aviña, 2013; Prasad, 2015). Os fatores de risco de DCV dentro do ambiente inflamatório da AR alteram a estrutura, propriedades e função da parede arterial e prejudicam a função endotelial (Hannawi, 2007; Hannawi, 2009; Hannawi, 2018).

Ademais, nos casos de comorbidades associadas, tais como obesidade, síndrome metabólica e hipertensão arterial sistêmica, compreende-se que as mesmas são alarmantes para o aumento do risco cardiovascular, fazendo com que episódios de infarto agudo do miocárdio, acidente vascular cerebral, tromboembolismo pulmonar e outras tromboses arteriais se tornem mais frequentes em pacientes acometidos por COVID-19 (Singer et al., 2017). Logo, o alto risco cardiovascular apresentado pela paciente, assim como o controle inadequado da atividade da AR, em associação com a infecção pelo COVID-19 pode ter sido o gatilho para o desenvolvimento da síndrome coronariana aguda e da trombose arterial de ilíaca esquerda.

É sabido que a lesão miocárdica associada à infecção pelo SARS-CoV-2 está incluída em um dos critérios de gravidade para a doença, segundo a última atualização do CDC (CDC, 2020), assim, infere-se que, da mesma forma que foi percebido pelos estudos realizados na cidade chinesa de Wuhan, a letalidade associada a essa condição corrobora para um maior determinismo de desfechos desfavoráveis, fazendo com que seja imprescindível a maior atenção com os sinais e sintomas apresentados por pacientes de alto risco cardiovascular, com objetivo de detectar quadros agudos e atuar precocemente neles, como podemos observar no caso da paciente que foi abordada em tempo de tratamento do infarto agudo do miocárdio, assim como a destreza em detectar e tratar precocemente a trombose arterial em membro inferior esquerdo.

Na resposta inflamatória sistêmica provocada pela COVID-19, observam-se concentrações mais altas de citocinas que estão relacionadas à injúria do sistema cardiovascular. O aumento de troponina é acompanhado de elevação de outros marcadores inflamatórios, como D-dímero, ferritina, interleucina-6 (IL-6), desidrogenase láctica (DHL), proteína C reativa, procalcitonina e contagem de leucócitos. Compreende-se que os pacientes que necessitam de terapia intensiva, na maioria das vezes, apresentam elevação desses marcadores associada a um intenso desconforto respiratório (Costa et al., 2020).

\section{Considerações Finais}

Em síntese, apesar da paciente em estudo não apresentar síndrome respiratória aguda, a presença constante de marcadores inflamatórios elevados, principalmente D-dímero, e a associação com o alto risco cardiovascular prévio indicaram manutenção do tratamento com anticoagulantes injetáveis e orais.

A esperança para o término dessa terrível pandemia que assombra o mundo é a vacinação em massa da população, entretanto é fundamental lembrar que, embora as vacinas possam ajudar a acabar com a pandemia, elas não resolverão tudo. À medida que a crise da COVID-19 continuar, ainda será necessárias medidas para evitar disseminação do vírus e, consequentemente, mais mortes. É preciso seguir e adotar uma abordagem do tipo "faça tudo", incluindo as medidas de proteção: lavar as mãos frequentemente com água e sabão ou álcool em gel e cobrir a boca com o antebraço quando tossir ou espirrar (ou utilize um lenço descartável e, após tossir/espirrar, jogue-o no lixo e lave as mãos). É importante manter-se pelo menos 1 metro de distância das outras pessoas. Quando o distanciamento físico não é possível, o uso de uma máscara também é uma medida de proteção. A nível individual, essas medidas de proteção funcionam inclusive contra as novas variantes identificadas até o momento. 


\section{Referências}

Aviña-Zubieta, J. A., Abrahamowicz, M., De Vera, M. A., Choi, H. K., Sayre, E. C., Rahman, M. M., Sylvestre, M. P., Wynant, W., Esdaile, J. M., \& Lacaille, D. (2013). Immediate and past cumulative effects of oral glucocorticoids on the risk of acute myocardial infarction in rheumatoid arthritis: a population-based study. Rheumatology. 52(1):68-75. 10.1093/rheumatology/kes353.

Bergström, L. T., \& Turesson, J. C. (2009). Cardiovascular morbidity and mortality remain similar in two cohorts of patients with long-standing rheumatoid arthritis seen in 1978 and 1995 in Malmö, Sweden. Rheumatology. 48:1600-5.

Bichile, T., \& Petri, M. (2014). Prevention and management of comorbidities in SLE. Presse Med. 43(6):187-95. 10.1016/j.lpm.2014.03.009.

Burner, T. W., \& Rosenthal, A. K. (2009). Diabetes and rheumatic diseases. Curr Opin Rheumatol. 21(1):50-4. 10.1097/BOR.0b013e32831bc0c4.

Coronavirus Disease (COVID-19) - World Health Organization. https://www.who.int/emergencies/diseases/novel-coronavirus-2019.

Coronavirus Disease 2019. Centers for Disease Control and Prevention, 25 de junho de 2020, https://www.cdc.gov/media/releases/2020/p0625-updateexpands-covid-19.html.

Costa, I. B. S. S., Bittar, C. S., Rizk, S. T., Araújo Filho, A. E., Santos, K. A. Q., Machado, T. I. V., Andrade, F. T. A., González, T. B., Arévalo, A. N. G., Almeida, J. P., Bacal, F., Oliveira, G. M. M., Lacerda, M. V. G., Barberato, S. H., \& Chagas, A. C. P. (2020). O Coração e a COVID-19: O que o Cardiologista Precisa Saber. Arquivos Brasileiros de Cardiologia. 114(5):805-16. 10.36660/abc.20200279.

Definição de Caso e Notificação. https://coronavirus.saude.gov.br/definicao-de-caso-e-notificacao.

Fernández, A. C., Izquierdo, A., Subirana, I., Farré, N., Vila, J., Durán, X., Guimaraes, M. G., Valdivielso, S., Cabero, P., Soler, C., Ribas, C. G., Rodríguez, C., Llagostera, M., Mojón, D., Vicente, M., González, E. S., Carpintero, A. S., Tevar, C., Marrugat, J., \& Vaquerizoa, B. (2020).

Gabriel, S. E., \& Cynthia, S. (2012). Crowson. Risk Factors for Cardiovascular Disease in Rheumatoid Arthritis. Current Opinion in Rheumatology. 24(2):171-6. 10.1097/BOR.0b013e32834ff2fd.

Gomes, A. A. Estudo de caso - planejamento e métodos. Nuances: estudos sobre Educação. Presidente Prudente, SP, ano XIV, 15(16), 215-221, jan./dez. 2008.

Figueiredo Neto, J. A., Braga, F. G. M., Moura, L. Z., Figueiredo, M. A. S., Figueiredo, V. M. S., Rocha, R. M., \& Mesquita, E. T. Doença de Coronavírus-19 e o Miocárdio. Arquivos Brasileiros de Cardiologia. 114(6):1051-57. 10.36660/abc.20200373.

Filippini, F. B., Silva, R. L., Vieira, R. G., Fattah, T., \& Joaquim, R. M. (2020). Infarto agudo do miocárdio tipo 2 induzido por COVID-19 em paciente com doença arterial coronariana grave desconhecida. J Transcat Intervent, 28(9). 10.31160/JOTCI202028A20200022.

Folha informativa COVID-19 - Escritório da OPAS e da OMS no Brasil - OPAS/OMS. Organização Pan-Americana da Saúde. https://www.paho.org/pt/covid19.

Hannawi, S., Haluska, B., Marwick, T. H., \& Thomas, R. (2007). Atherosclerotic Disease Is Increased in Recent-Onset Rheumatoid Arthritis: A Critical Role for Inflammation. Arthritis Research \& Therapy. 9(6):116. 10.1186/ar2323.

Hannawi, S., Hannawi, H., Alokaily, F., Naredo, E., Moller, I., \& Salmi, I. A. (2018). Recent-Onset of Rheumatoid Arthritis Leads to Increase in Wall Thickness of Left Anterior Descending Coronary Artery. An Evidence of Subclinical Coronary Artery Disease. Saudi Medical Journal. 39;(12):1213-17. 10.15537/smj.2018.12.23185.

Hannawi, S., Marwick, T. H., \& Thomas, R. (2009). Inflammation predicts accelerated brachial arterial wall changes in patients with recent-onset rheumatoid arthritis. Arthritis Research \& Therapy. 11(2):51. 10.1186/ar2668.

Lazaros, G., \& Dimitrios, T. (2015). Rheumatoid Arthritis and Atherosclerosis: Could Common Pathogenesis Translate Into Common Therapies?. Hellenic J Cardiol., 56(5):414-7. https://pubmed.ncbi.nlm.nih.gov/26429370/.

Markers of myocardial injury in the prediction of short-term COVID-19 prognosis. Revista Espanola De Cardiologia. 5:811-818. PubMed, 10.1016/j.recesp.2020.09.017.

McInnes, I. B., \& Scheet, G. (2011). The Pathogenesis of rheumatoid arthritis. N Engl J Med. 365;(2):2205-19.

Mikuls, T. D., Johnson, S. R., Gravallese, E. M., \& Saag, K. G. (2020). Coronavirus disease 2019 (COVID-19): Care of patients with systemic rheumatic disease during the pandemic. 29 Mai 292020 [citado em 05 Jun 2020]. In: UpToDate [Internet]. 2020. https://www.uptodate.com/contents/coronavirusdisease-2019-covid-19-care-of-patients-with-systemic-rheumatic-disease-during-the-pandemic.

Mikuls, T. R. (2003). Comorbidity in rheumatoid arthritis. Best Pract Res Clin Rheumatol. 17(5):729-52. 10.1016/s1521-6942(03)00041-x.

Mikuls, T. R., Johnson, S. R., Fraenkel, L., Arasaratnam, R. J., Baden, L. R., Bermasm B. L., Chatham, W., Cohen, S., Costenbader, K., Gravallese, E. M., Kalil, A. C., Weinblatt, M. E., Winthrop, K., Mudano, A. S., Turner, A., \& Saag, K. G. (2020). American college of rheumatology guidance for the management of rheumatic disease in adult patients during the covid-19 pandemic: version 1. Arthritis \& Rheumatology. 2(0):1-11.

Nurmohamed, M. T., Heslinga, M., \& Kitas, G. D. (2015). Cardiovascular comorbidity in rheumatic diseases. Nat Rev Rheumatol. 11:693-704.

Prasad, M., Hermann, J., Gabriel, S. E., Weyand, C. M., Mulvagh, S., Mankad, R., Oh, J. K., Matteson, E. L., \& Lerman, A. Cardiorheumatology: Cardiac Involvement in Systemic Rheumatic Disease. Nature Reviews Cardiology. 12(3):168-76. 10.1038/nrcardio.2014.206.

Richardson, S., Hirsch, J. S., Narasimhan, M., Crawford, J. M., McGinn, T., \& Davidson, K. W. (2020). Presenting characteristics, comorbidities, and outcomes among 5700 patients hospitalized with COVID-19 in the New York City area. JAMA. 323(20):2052-2059. 
Research, Society and Development, v. 10, n. 14, e456101422277, 2021

(CC BY 4.0) | ISSN 2525-3409 | DOI: http://dx.doi.org/10.33448/rsd-v10i14.22277

Sarkisian, L. (2016). Prognostic Impact of Myocardial Injury Related to Various Cardiac and Noncardiac Conditions. The American Journal of Medicine. 129(5):506-514. 10.1016/j.amjmed.2015.12.009.

Singer, M., Bulled, N., Ostrach, B., \& Mendenhall, E. (2017). Syndemics and the biosocial conception of health. Lancet. 389;(4):941-950. 10.1016/S01406736(17)30003-X.

Solomon, D. H., Kremer, J., Curtis, J. R., Hochberg, M. C., Reed, G., Tsao, P., Farkouh, M. E., Setoguchi, S., \& Greenberg, J. D. (2010). Explaining the cardiovascular risk associated with rheumatoid arthritis: traditional risk factors versus markers of rheumatoid arthritis severity. Ann Rheum Dis. 69:1920-5.

Wu, C., Chen, X., Cai, Y., Xia, J., Zhou, X., \& Xu, S. (2020). Risk factors associated with acute respiratory distress syndrome and death in patients with coronavirus disease 2019 pneumonia in Wuhan, China. JAMA Intern Med. 180(7):934-943. https://www.doi.org/10.1001/jamainternmed.2020.0994.

Yang, J., Zheng, Y., Gou, X., Pu, K., Chen, Z., Guo, Q., Ji, R., Wang, H., Wang, Y., \& Zhou, Y. (2020). Prevalence of comorbidities in the novel Wuhan coronavirus (COVID-19) infection: a systematic review and meta-analysis. Int J Infect Dis. 94:91-5.

Zhou, F., Yu, T., Du, R., Fan, G., Liu, Y., Liu, Z., Xiang, J., Wang, Y., Song, B., Gu, X., Guan, L., Wei, Y., Li, H., Wu, X., Xu, J., Tu, S., Zhang, Y., Chen, H., \& Cao, B. (2020). Clinical course and risk factors for mortality of adult in patients with COVID-19 in Wuhan, China: a retrospective cohort study. Lancet. 395(2):1054-62.

Zhu, N., Zhang, D., Wang, W., Li, X., Yang, B., Song, J., Zhao, X., Huang, B., Shi, W., Lu, R., Niu, P., Zhan, F., Ma, X., Wang, D., Xu, W., Wu, G., Gao, G. F., \& Tan, W. (2020). A novel coronavirus from patients with pneumonia in China, 2019. N Engl J Med.; 382:727-33. 